\title{
Efecto de metales pesados en el crecimiento de la microalga tropical Tetraselmis chuii (Prasinophyceae)
}

\author{
Jiudith Cordero ${ }^{1}$, Miguel Guevara ${ }^{1}$, Ever Morales $^{2} \&$ César Lodeiros ${ }^{1 *}$ \\ 1 Instituto Oceanográfico de Venezuela, Universidad de Oriente, Cumaná 6101, Venezuela \\ 2 Departamento de Biología, Facultad de Ciencias de la Universidad del Zulia, Maracaibo, Venezuela \\ *Autor de correspondencia: Tel.: 093 302118; Tel./fax: 315902; clodeiro@sucre.udo.edu.ve.ve \\ Recibido 03-IX-2001. Corregido 11-II-2004. $\quad$ Aceptado 18-VII-2005.
}

\begin{abstract}
Efect of heavy metals on the growth of the tropical microalga Tetrasermis chuii (Prasinophyceae). We determined the toxic effect of four metals, cadmium $(\mathrm{Cd})$, copper $(\mathrm{Cu})$, mercury $(\mathrm{Hg})$ and lead $(\mathrm{Pb})$, on the tropical microalga Tetraselmis Chuii (Butcher, 1959). We exposed $50 \mathrm{ml}$ of cultivated microalgae (f/2 Guillard) in the exponential growth phase, with three replicates, to concentrations of 0 (control), 0.1, 1.0, 5.0, 10.0 and $20.0 \mathrm{mg} \cdot \mathrm{l}^{-1}$ with each metal for $96 \mathrm{hr}$. We evaluated the lethal effect daily, through the cellular count. In the control treatment (not exposed to any metal) we observed an increase in cellular density. In all treatments exposed to metals, we observed a decrease in cellular density, which accelerated in $48 \mathrm{~h}$, after which it became less pronounced. There were exceptions with low concentrations of $\mathrm{Cd}$ and $\mathrm{Cu}$ at $24 \mathrm{~h}$, as there was no significant decrease, probably due to their use as micronutrients at these low concentrations. The metal that caused the most lethal effect was $\mathrm{Pb}$, which killed $50 \%$ of the microalgal population at a concentration of $0.40 \mathrm{mg} \cdot \mathrm{l}^{-1}$. This concentration was 3 times lower than that of mercury and 13 times lower than those of cadmium and copper. The microalga Tetraselmis chuii is recommended as a model species to estimate the toxic effects of xenobiotics on tropical seawater environments. Rev. Biol. Trop. 53(3-4): 325-330. Epub 2005 Oct 3.
\end{abstract}

Key words: Heavy metals, contamination, Tetraselmis chuii, toxicity, phytoplankton.

El medio acuático y en especial el marino es uno de los ambientes más expuestos a los contaminantes, debido a que las descargas, sean por vía terrestre, acuático-terrestre o atmosférica, tienen como receptáculo final el ambiente marino (Acuña-Gonzalez et al. 2004, Coll et al. 2004, García-Céspedes et al. 2004, Sponberg 2004a,b,c, Norville 2005, Rojas de Astudillo et al. 2005). En estos sistemas las microalgas constituyen el principal componente del fitoplancton que soporta la cadena trófica, de esta manera, un cambio, sea cualitativo o cuantitativo, producido por un contaminante, podría repercutir drásticamente en el ecosistema (Nalewajko y Olavenson 1998, Franklin et al. 2000).

A concentraciones bajas, muchos de los metales juegan un papel esencial en el metabolismo de las microalgas, tal es el caso de los metales Zn y Cu (Tadros et al. 1990); sin embargo, cuando las concentraciones son altas, dan origen a condiciones de contaminación del medio que provocan desbalances metabólicos en el fitoplancton (Rodríguez y Rivera 1995). Por ejemplo, cuando el fitoplancton es expuesto a concentraciones elevadas de metales pesados, se produce una inhibición del crecimiento, así como cambios morfológicos, generados como una respuesta fisiológica a la exposición del metal que tienen como consecuencia una menor capacidad de respuesta de las poblaciones al ambiente (Rand y Petrocelli 1985, Walsh et al. 1987, Visviki y Rachlin 1994).

Algunas microalgas de zonas templadas han sido consideradas como especies modelos 
para estudiar efectos de contaminación, debido a su sensibilidad ante diversos materiales de prueba; además de ser conocido sus requerimientos nutricionales, de poseer una alta tasa de crecimiento y de ser cultivadas fácilmente en el laboratorio (Couture et al. 1985, Nalewajko y Olavenson 1998). No obstante, pocas especies tropicales han sido estudiadas.

El presente trabajo evalúa el efecto tóxico de diversos metales sobre la especie Tetraselmis chuii, una microalga prasinofícea, de forma cilíndrica, de unos 8 a $16 \mu \mathrm{m}$ de longitud y que debido a su gran distribución en los ecosistemas tropicales y amplia utilización en cultivos de laboratorios, le confiere una importancia notable como una posible especie modelo para estimar el impacto ambiental producido por xenobióticos.

\section{MATERIALES Y MÉTODOS}

El efecto del cadmio $\left(\mathrm{CdCl}_{2}, 81 \%\right.$ de pureza), cobre $\left(\mathrm{CuSO}_{4} 5 \mathrm{H}_{2} \mathrm{O}, 99 \%\right.$ de pureza), mercurio $\left(\mathrm{HgCl}_{2}, 99.55 \%\right.$ de pureza $)$ y plomo $\left(\mathrm{PbCl}_{2}, 99 \%\right.$ de pureza ) sobre cultivos, en fase logarítmica, de la microalga Tetraselmis chuii (Butcher 1959), proveniente del cepario del Lab. Acuicultura, extensión Plancton, Instituto Oceanográfico de Venezuela, de la Universidad de Oriente, se evaluó a las concentraciones de 0.0 (control); $0.1 ; 1.0 ; 5.0 ; 10.0 ;$ y $20.0{\mathrm{mg} \cdot \mathrm{l}^{-1}}^{-1}$ para cada metal.

El bioensayo comenzó a partir de la inoculación de 30000 células $\mathrm{ml}^{-1}$ de la microalga en $800 \mathrm{ml}$ del cultivo f/2 de Guillard (utilizando agua de mar proveniente de la zona de Turpialito, Golfo de Cariaco, Venezuela, excenta de focos contaminantes), contenidos en matraces de 11 y por triplicado. Cuando los cultivos alcanzaron la fase logarítmica a una densidad

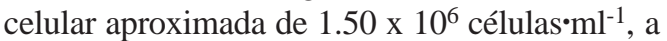
cada cultivo se le extrajo una alícuota de $50 \mathrm{ml}$, la cual se depositó en un matraz de 100 ml para cada concentración de cada metal analizado. Todo el bioensayo fue controlado en cuanto a temperatura $\left(25 \pm 1{ }^{\circ} \mathrm{C}\right)$, iluminación continua (2000 lux), salinidad (36\%o), estableciendo una agitación manual diaria.
La densidad celular fue determinada mediante el recuento de las microalgas utilizando una cámara de Neubauer, a partir de muestras de un $\mathrm{ml}$, tomadas diariamente hasta 96 h. En dicho recuento, no se cuantificaron células no pigmentadas o lisadas, considerandas éstas como células muertas. En función de minimizar el error generado por la variabilidad del número celular de las réplicas, la evaluación se estableció transformando los datos en porcentaje de incremento o decrecimiento celular con respecto a la densidad inicial de cada réplica. Un análisis de varianza de una vía, previa transformación de los datos a arcoseno, en función de normalizar los datos (Zar 1984), fue aplicado, para determinar diferencias significativas $(P<0.05)$ de la densidad microalgal entre los metales en cada concentración e intervalo de tiempo analizado. En aquellos casos donde existieron diferencias significativas $(P<0.05)$, se aplicó la prueba de comparaciones múltiples de Scheffé. De igual manera, un análisis similar se aplicó para cada metal, en función de determinar diferencias entre los recuentos establecidos diariamente.

Por otra parte, el efecto de cada metal se analizó mediante la evaluación de la concentración letal del $50 \%$ de la población del cultivo $\left(\mathrm{CL}_{50}\right)$, utilizando un programa computarizado (Stephan 1977) siguiendo los protocolos estándares para bioensayos de toxicidad con especies acuáticas (Esclapés 1999). Los métodos binominal, probit, promedio movil y logit fueron evaluados y se escogió el de mejor ajuste (probit).

\section{RESULTADOS}

En general, mientras se evidenció un crecimiento de los cultivos de la microalga sin exposición a metales (control), se observó una significativa $(P<0.05)$ disminución de la densidad algal en cada uno de los tratamientos con exposición a los metales, con excepción de los cultivos expuestos a 0.1 y $1.0 \mathrm{mg} \cdot \mathrm{l}^{-1}$ de $\mathrm{Cd}$ y $\mathrm{Cu}$, a las $24 \mathrm{hr}$, donde la disminución de la densidad algal fue no significativa $(P>0.05)$ con respecto a la inicial (Fig. 1). Un caso similar 
ocurrió con el $\mathrm{Hg}$, pero sólo a la concentración de $0.1 \mathrm{mg} \cdot \mathrm{l}^{-1}$.

Todos los metales ensayados produjeron una tendencia de decrecimiento acelerado hasta las $48 \mathrm{hr}$, evidenciándose una letalidad cercana al $50 \%$, para todos los tratamientos a partir de
$5 \mathrm{mg}^{-1} \mathrm{l}^{-1}$ (Fig. 1). Esta letalidad fue menos pronunciada después de las $48 \mathrm{hr}$.

La concentración letal al 50\% de la población microalgal $\left(\mathrm{CL}_{50}\right)$ fue mayor para las microalgas expuestas al $\mathrm{Pb}$, con una relación de efecto letal de $\mathrm{Pb}>\mathrm{Hg}>\mathrm{Cd}>\mathrm{Cu}$, donde la concentración
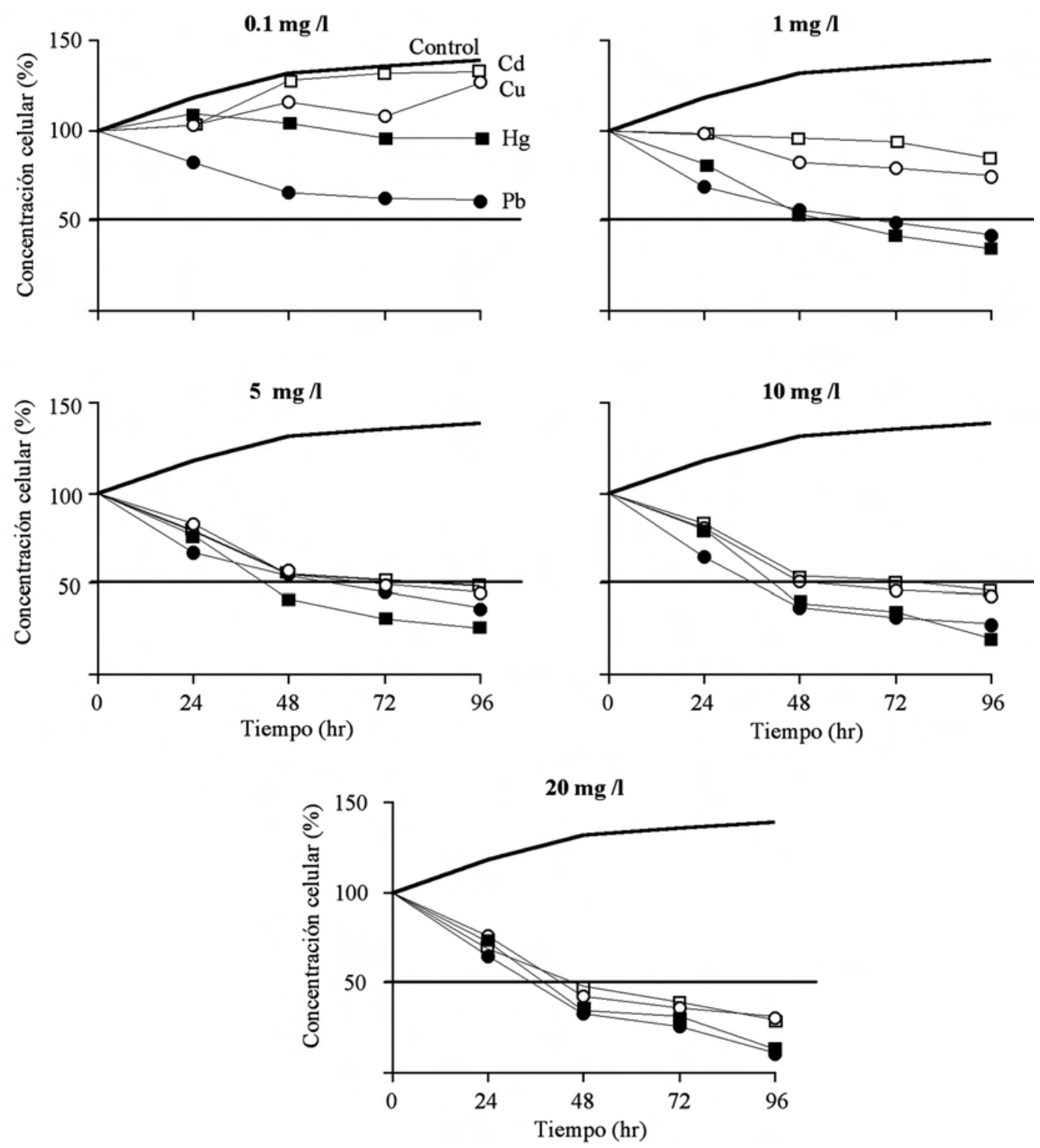

Fig. 1. Efecto del Cd ( $\square), \mathrm{Cu}(\mathrm{O}), \mathrm{Hg}(\boldsymbol{\square}) \mathrm{Pb}(\bullet)$ a las concentraciones de $0.1 ; 1 ; 5 ; 10$ y $20 \mathrm{mg} / \mathrm{l}$ sobre cultivos en fase logarítmica de la microalga Tetraselmis chuii.

Fig.1. Effect of $\mathrm{Cd}, \mathrm{Cu}, \mathrm{Hg}$ y $\mathrm{Pb}$ to the concentration of $0.1 ; 1 ; 5 ; 10$ y $20 \mathrm{mg} / \mathrm{l}$ on logaritmic phase culture of microalgae Tetraselmis chuii. 
del $\mathrm{Pb}\left(0.4 \mathrm{mg} \cdot \mathrm{l}^{-1}\right)$ fue casi 3 veces menor que la establecida para el $\mathrm{Hg}\left(1.1 \mathrm{mg}^{\cdot{ }^{-1}}\right)$ y más de 13 veces menor que la del $\mathrm{Cd}\left(5.4 \mathrm{mg}^{\cdot l^{-1}}\right)$ y $\mathrm{Cu}$ $\left(6.4 \mathrm{mg}^{-1}{ }^{-1}\right)$ (Cuadro 1).

\section{DISCUSIÓN}

Todos los metales ensayados a las diferentes concentraciones tuvieron un efecto letal en la microalga Tetraselmis chuii, a excepción del tratamiento con exposición al $\mathrm{Cu}$ y el $\mathrm{Cd}$ a la concentración menor ensayada $\left(0.1 \mathrm{mg} \cdot \mathrm{l}^{-1}\right)$, los cuales si bien proporcionaron densidades celulares menores al control (sin exposición al metal), produjeron, una mayor densidad celular que la inicial del bioensayo, sugiriendo una activa división celular. Este comportamiento, puede estar relacionado con la utilización del $\mathrm{Cu}$ como micronutriente (Rodríguez y Rivera 1995). En el caso del Cd, aunque este metal no ha sido establecido como micronutriente, estudios recientes (Cullen et al. 1999, Sunda y Huntsman 2000) han considerado que puede actuar como tal cuando se encuentra en concentraciones trazas y existe limitación de algunos micronutriente esenciales; situación que probablemente fue establecida en los cultivos ensayados, ya que el bioensayo se estableció con cultivos en una fase logarítmica avanzada, donde gran parte de los elementos trazas del medio de cultivo, probablemente se encontraban consumidos. Estos resultados, con respecto al Cd, son acordes con Romero (1999), quien en un estudio realizado con Tetraselmis sp. y Chaetoceros sp. encontró que la disminución del crecimiento poblacional de estas microalgas ante el $\mathrm{Cd}$ se manifiesta a partir de concentraciones superiores a $4.0 \mathrm{mg} \cdot \mathrm{l}^{-1}$.

Con la excepción del $\mathrm{Cu}$ y $\mathrm{Cd}$ y en concentraciones superiores a $0.1 \mathrm{mg}^{\cdot l^{-1}} 1$, se observó un decrecimiento acelerado hasta las $48 \mathrm{hr}$, a partir de cuando la proporción de letalidad se hace menos pronunciada, posiblemente, debido a adaptaciones de las células como producto de la bioacumulación de los metales a través de procesos antitóxicos. En este sentido, es importante señalar, que se observó una tendencia del aumento del volumen celular a medida que transcurría el tiempo de exposición y concentración de los metales (datos no mostrados). Observaciones similares fueron informadas por Rereiz et al. (1994) en cultivos de la diatomea Phaeodactylum tricornutum expuestos a diferentes concentraciones de $\mathrm{Cu}$. Estudios sobre la fisiología y ultraestructura de la microalga con la determinación de procesos enzimáticos de síntesis de proteínas relativas a procesos antitóxicos, tipo metalotioninas, son necesarios para dilucidar dicha hipótesis.

El metal que produjo mayor efecto fue el $\mathrm{Pb}$, seguido del $\mathrm{Hg}$, con los cuales se obtuvieron una concentración letal al $50 \%$ de la población en los cultivos de Tetraselmis chuii, muy superior a las ejercidas por el $\mathrm{Cu}$ y el $\mathrm{Cd}$. Esto corrobora la mayor letalidad del $\mathrm{Pb}$ y $\mathrm{Hg}$, dos metales no esenciales para el crecimiento microalgal.

Aunque la mayoría de los estudios de efectos tóxicos agudos en organismos acuáticos se realizan como una respuesta a períodos de 96 hr (Esclapés 1999), la condición de reproducción rápida de las microalgas y el

CUADRO 1

Concentración letal al 50\% de los metales $\mathrm{Cd}, \mathrm{Cu}, \mathrm{Hg}$ y $\mathrm{Pb}$ en la microalga Tetraselmis chuii

TABLE 1

Lethal concentration at $50 \%$ of the heavy metal $\mathrm{Cd}, \mathrm{Cu}, \mathrm{Hg}$ and $\mathrm{Pb}$ in the microalgae Tetraselmis chuii

\begin{tabular}{lccc} 
Metal & $\mathrm{CL}_{50}(\mathrm{mg} / \mathrm{l})$ & \multicolumn{2}{c}{ Intervalo de Confianza al 95\% } \\
Límite Superior \\
Cadmio & & & \\
Cobre & 5.44 & 4.185 & 7.175 \\
Mercurio & 6.44 & 5.133 & 8.324 \\
Plomo & 1.14 & 0.053 & 4.582 \\
& 0.40 & 0.001 & 1.583
\end{tabular}


mayor efecto de los metales pesados de letalidad mostrada antes de las $48 \mathrm{hr}$, observada en el presente estudio, sugiere la reducción del tiempo de exposición de los metales en microalgas a $48 \mathrm{hr}$ para un protocolo de efectos agudos. La implementación de dicha reducción del tiempo de evaluación, permitiría menos costos de la prueba y una más rápida evaluación, evitando la posible adaptación microalgal a los metales, lo cual pudiera enmascarar el efecto tóxico del xenobiotico.

Debido al carácter amplio en la utilización de actividades de cultivo en zonas tropicales de Tetraselmis chuii, y características deseables para establecer pruebas de toxicidad como su volumen celular, el cual permite fácilmente realizar monitoreos con cámaras de recuento celular, universalmente utilizadas, así como la sensibilidad ante metales pesados mostrada en el presente estudio, permiten recomendarla como una especie modelo para la utilización en pruebas para estimar efectos tóxicos por xenobióticos en ambientes marinos tropicales.

\section{AGRADECIMIENTOS}

El presente estudio fue parcialmente financiado por el Departamento de Biología Pesquera del Instituto Oceanográfico de Venezuela de la Universidad de Oriente y el Fondo Nacional de Ciencia, Tecnología e Innovación de Venezuela (FONACIT).

\section{RESUMEN}

El presente trabajo determina el efecto tóxico de los metales cadmio $(\mathrm{Cd})$, cobre $(\mathrm{Cu})$, mercurio $(\mathrm{Hg})$ y plomo $(\mathrm{Pb})$ en la microalga tropical Tetraselmis chuii (Butcher, 1959). Se expuso, por triplicado, $50 \mathrm{ml}$ de cultivo (f/2 Guillard) de la microalga en fase de crecimiento logarítmica ante las concentraciones de 0 (control); $0.1 ; 1.0 ; 5.0$; 10.0 y $20.0 \mathrm{mg} \cdot \mathrm{l}^{-1}$ durante $96 \mathrm{hr}$. La evaluación del efecto letal se realizó diariamente, mediante recuento celular con una cámara de Neubauer. En el tratamiento control, sin exposición al metal, se observó un incremento de la densidad celular, en contraste con un decrecimiento en los tratamientos con exposición a los metales, los cuales fueron acelerados hasta las $48 \mathrm{hr}$, a partir de cuando el decrecimiento se hizo menos pronunciado. Una excepción se produjo con el $\mathrm{Cd}$ y el $\mathrm{Cu}$ a las $24 \mathrm{~h}$, donde no se determinó decrecimiento significativo, probablemente debido a su capacidad de actuar como micronutriente a bajas concentraciones. El metal que produjo mayor efecto fue el $\mathrm{Pb}$, produciendo una letalidad al $50 \%$ de la población microalgal a $0.40 \mathrm{mg} \cdot \mathrm{l}^{-1}$, la cual fue casi tres veces menor que la establecida para el $\mathrm{Hg}$ y más de 13 veces menor que la del $\mathrm{Cd} \mathrm{y} \mathrm{Cu}$. Se recomienda la microalga Tetraselmis chuii como especie modelo para la utilización en pruebas en función estimar efectos tóxicos por xenobióticos en el ambiente acuático marino tropical.

Palabras clave: metales pesados, contaminación, Tetraselmis chuii, toxicidad, fitoplancton.

\section{REFERENCIAS}

Acuña-González, J., J.A. Vargas-Zamora, E. GómezRamírez \& Jairo García-Céspedes. 2004. Hidrocarburos de petróleo, disueltos y dispersos, en cuatro ambientes costeros de Costa Rica. Rev. Biol. Trop. 52 (Suppl. 2): 43-50.

Cullen, J., T. Lane, F. Morel \& A. Sherrel. 1999. Modulation of $\mathrm{Cd}$ uptake in phytoplankton by seawater $\mathrm{CO}_{2}$ concentration. Nature 402: 165-166.

Couture, P., S. Visser, R. Vancoillie \& C. Blaise. 1985. Algal bioassays their significance in monitoring water quality with respect to nutrients and toxicants. Schweiz. Z. Hydrol. 47: 127-158.

Coll, M. \& J. Cortés \& D. Sauma. 2004. Características físico-químicas y determinación de plaguicidas en el agua de la laguna de Gandoca, Limón, Costa Rica. Rev. Biol. Trop. 52(Suppl. 2): 33-42.

Esclapés, M. 1999. Protocolos estándares para bioensayos de toxicidad con especies acuáticas y terrestres. Versión 2.0. Gerencia General de Tecnología, Departamento de Ecología y Ambiente. INTEVEP. Caracas. 215 p.

Franklin, N., J. Stauber, S. Markich \& R. Lim. 2000. pHdependent toxicity of copper and uranium to a tropical fresh water alga (Chlorella sp.). Aquat. Toxicol. 48: 275-289.

García-Céspedes, J., J. Acuña-González \& J.A. VargasZamora. 2004. Metales traza en sedimentos costeros de Costa Rica. Rev. Biol. Trop. 52(Suppl. 2): 51-60.

Nalewajko, C. \& M. Olavenson. 1998. Ecofisiological considerations in microalgal toxicity test, pp. 289-309. In P. Wells, K. Lee \& Ch. Blaise (eds). Microscale 
testing in aquatic toxicity: advances, technical and practice. CRC, Boca Raton, Florida.

Norville, W. 2005. Spatial distribution of heavy metals in sediments from the Gulf of Paria, Trinidad. Rev. Biol. Trop. 53(Suppl. 1): 33-40.

Rand, G. \& S. Petrocelli. 1985. Fundamentals of aquatic toxicology. Hemisphere Corporation, Washington, D.C. $665 \mathrm{p}$

Reiriz, S., A. Cid, E. Torres, J. Abalde \& C. Herrero. 1994. Different responses of the marine diatom Phaodactylum tricornutum to Koper toxicity. Microbiología SEM 10: 263-272.

Rodríguez, L. \& D. Rivera. 1995. Efecto del cobre y el cadmio en el crecimiento de Tetraselmis suecica (KYLIN) BUTCHER y Dunaliella salina TEODORESCO. Estud. Oceanol. 14: 61-74.

Rojas de Astudillo, L., I. Chang Yen \& I. Bekele. 2005. Heavy metals in sediments, mussels and oysters from Trinidad and Venezuela. Rev. Biol. Trop. 53(Suppl. 1): 33-40.

Romero, Y. 1999. Efecto del cadmio sobre las microalgas Tetraselmis sp. y Chaetoceros sp. Tesis de Licenciatura. Universidad de Oriente, Cumaná, Venezuela. $92 \mathrm{p}$.

Spongberg, A.L. 2004a. PCB contamination in surface sediments in the coastal waters of Costa Rica. Rev. Biol. Trop. 52(Suppl. 2): 1-10.
Spongberg, A.L. 2004b. PCB concentrations in sediments from the Gulf of Nicoya estuary, Pacific coast of Costa Rica. Rev. Biol. Trop. 52(Suppl. 2): 11-22.

Spongberg, A.L. 2004c. PCB contamination in marine sediments from Golfo Dulce, Pacific coast of Costa Rica. Rev. Biol. Trop. 52(Suppl. 2): 23-32.

Stephan, C.E. 1977. Methods for calculation an LC50. Pp. 65-84. In F.L. Mayer \& J.L. Hamelink (eds). Aquatic toxicology and hazaed evaluations. ASTMSTP534. American Society for Testing Materials, Filadelfia, Pensilvania, EEUU.

Sunda, W. \& S. Huntsman. 2000. Effect of Zn, Mn and Fe on $\mathrm{Cd}$ accumulation in phytoplankton: Implications for oceanic Cd cyclin. Limn. Oceanogr. 45: 1501-1516.

Tadros, M., P. Bulthia \& W. Smith. 1990. Diferential response of marine diatoms to trace metal. Bull. Environ. Contam. Toxicol. 44: 826-831.

Visviki, Y. \& W. Rachlin. 1994. Acute and chronic exposure of Dunaliella salina and Chlamydomonas bullosa to copper and cadmium: Effects on ultrastructure. Archv. Environ. Contam. Toxicol. 26: 1554-1562.

Walsh, G., M. Yoder, L. Laughlin \& E. Lores. 1987. Responses of marine unicellular algae to brominated organic compound in six growth media. Ecotox. Envir. Safety 14: 215-222.

Zar, J. 1984. Biostatical analysis. Prentice Hall, Nueva Jersey, EEUU. 699 p. 